\title{
DAS ÖSTERREICHISCHE BUNDES-VERFASSUNGSGESETZ (1920) UND DIE WEIMARER REICHSVERFASSUNG (1919). WEIMARS NEGATIVER EINFLUSS AUF DIE ÖSTERREICHISCHE VERFASSUNG
}

\section{THE AUSTRIAN FEDERAL CONSTITUTION (1920) AND THE WEIMAR CONSTITUTION (1919). THE HARMFUL INFLUENCE OF WEIMAR ON THE AUSTRIAN CONSTITUTION}

\author{
András Jakab \\ Universität Salzburg
}

\begin{abstract}
SUMARIO: I. GEBURT DES ÖSTERREICHISCHEN BUNDESVERFASSUNGSGESETZES IM SCHATTEN VON WEIMAR.- II. DIE PARTIELLE WEIMARISIERUNG DES B-VG IN 1929.- III. UNTERSCHIEDE ZWISCHEN DEM B-VG NACH 1929 UND DER WRV.- IV. ZWEI UNTERGANGSGESCHICHTEN.- V. ERBE.
\end{abstract}

Resumen: Das österreichische Bundes-Verfassungsgesetz (B-VG, 1920) wurde um ein Jahr später verabschiedet als die Weimarer Reichsverfassung (WRV, 1919). Während der Verabschiedung beobachtete man sorgfältig die verfassungsrechtlichen Entwicklungen in Deutschland, und an einigen Stellen (vor allem bezüglich der bundesstaatlichen Kompetenzordnung) wurden sogar Textstellen übernommen. Den wichtigsten Einfluss auf das B-VG hat die WRV 1929 ausgeübt, als die bis dahin eindeutig parlamentarische Regierungsform des B-VG nach Weimarer Muster in eine halb-präsidentielle umgewandelt wurde. Diese Änderung war sowohl demokratietheoretisch, als auch verfassungspolitisch eine eindeutige Verschlechterung des ursprünglichen Konzeptes von 1920. Jedoch prägt diese Änderung bis heute das österreichische Modell.

Abstract: The Austrian Federal Constitution (1920) was adopted one year later than the Weimar Constitution. The constitutional developments in Germany were carefully followed during the Austrian constitution-making process, and at certain points (especially concerning the distribution of competences between the Bund and the Länder) the German text was borrowed by the Austrian constitution-maker. The most important influence, however, happened in 1929 when (inspired by the Weimar Constitution) a semi-presidential system was introduced in Austria instead of the original (1920) pure parliamentary system. This change was both from a democracy theoretical and a practical constitutional-political perspective a deterioration that has effects until today on the Austrian constitutional system.

Palabras clave: parlamentarismo, semipresidencialismo, autoritarismo, teorias de democracia, erosión de la democracia. 
Key Words: parliamentarism, semi-presidential systems, authoritarian regimes, theories of democracy, erosion of democracy.

\section{GEBURT DES ÖSTERREICHISCHEN BUNDES-VERFASSUNGSGESETZES IM SCHATTEN VON WEIMAR}

Die bis heute wirkungsvollsten Verfassungen der Periode unmittelbar nach dem Ersten Weltkrieg sind die Weimarer Reichsverfassung (1919, im Weiteren: WRV) und das österreichische Bundes-Verfassungsgesetz (1920, im Weiteren: BVG). ${ }^{1}$ Am Beginn beider Verfassungen stand ein starkes Legitimationsdefizit in der eigenen Bevölkerung: Sie waren Symbole für den Verlust des Weltkrieges und standen stellvertretend für die nationale Demütigung durch die Friedensverträge. Sie hatten auch beide einen Kompromisscharakter: Keine der politischen Kräfte war völlig mit ihnen zufrieden. ${ }^{2}$ Das B-VG wurde mehr als ein Jahr später verabschiedet als die WRV, und in den Debatten zum B-VG wurde ausdrücklich auf die Regeln der WRV hingewiesen (einige Formulierungen der bundesstaatlichen Kompetenzartikel wurden sogar wortwörtlich übernommen). ${ }^{3}$ Dies wurde auch von den Juristen, die an der textlichen Fassung gearbeitet haben, offen zugegeben: „Die österreichische Bundesverfassung hat sich im allgemeinen mehr die deutsche Reichsverfassung als die schweizerische und die amerikanische Bundesverfassung zum Vorbild genommen."4

Dies geschah nicht nur deswegen, weil die WRV vor dem B-VG verabschiedet wurde, und nicht nur wegen der sprachlichen und kulturellen Nähe, sondern auch weil man in Wien auf einen möglichst baldigen Anschluss an den größeren Bruder gehofft hat. ${ }^{5}$ Diese Tendenz galt nicht nur für die deutschnationalen,

\footnotetext{
1 Für wertvolle Literaturhinweise, kritische Lektüre bzw interessante Diskussionen zum Thema bin ich Lando Kirchmair, Valerie Schwarzer und Ewald Wiederin dankbar.

2 Stefanie Vielgut, Demokratie, Verfassung und Rechtspositivismus. Über die Entstehung und den Untergang der Weimarer Republik und der Ersten Republik Österreich, AV Akademiker Verlag, 2012 , p. 34.

3 Felix Ermacora, Die Entstehung der Bundesverfassung 1920. Band II, Braumüller, Wien, 1989, pp. 74, 76 und 78; Georg Schmitz, „Vorentwürfe von Hans Kelsen für die österreichische Bundesverfassung“, Österreichische Juristen-Zeitung 1979, pp. 85 et seq. mit Hinweisen insb. auf die Regelung von Untersuchungsausschüssen (Art 34 WRV) und der Bundesstaatlichkeit (Art 61-67, 69, 74 WRV). Auch Art 17 WRV über das Verhältniswahlsystem mit allgemeinem, gleichem, unmittelbarem und geheimem Wahlrecht für sämtliche allgemeinen Vertretungskörper wurde fast wortwörtlich übernommen (vor allem Art 26 B-VG), siehe Ewald Wiederin, „Die Weimarer Reichsverfassung im internationalen Kontext", in: Horst Dreier / Christian Waldhoff (Hrsg), Das Wagnis der Demokratie. Eine Anatomie der Weimarer Reichsverfassung, Beck, München, 2018, p. 59.

${ }^{4}$ Hans Kelsen / Adolf Merkl / Georg Froehlich, Die Bundesverfassung vom 1. Oktober 1920, Deuticke, Wien, 1922, p. 101.

5 Christian Sima, Österreichs Bundesverfassung und die Weimarer Reichsverfassung. Der Einfluss der Weimarer Reichsverfassung auf die österreichische Verfassung 1920 bis 1929, Lang, Frankfurt am Main, 1993, p. 7: „In Art 2 des Gesetzes über die Staats- und Regierungsform vom 12. November 1918 wurde Deutschösterreich zu einem Teil der Deutschen Republik erklärt. Durch Art 88 des Staatsvertrages von St. Germain wurde aber der Anschluß an Deutschland untersagt, und zwar mindesten solange, bis der Völkerbund einem solchen seine Zustimmung erteilt."
} 
sondern auch für die sozialdemokratischen Kräfte (Weimar war ja am Anfang sozialdemokratisch geprägt). ${ }^{6}$

Die zwei wichtigsten Unterschiede zwischen den Texten betrafen einerseits die Grundrechte, andererseits die Stellung des Staatsoberhauptes. In Österreich konnte man sich wegen der starken ideologischen Kontroversen nach dem Ersten Weltkrieg auf keinen neuen Grundrechtskatalog einigen. Deshalb behielt man auch das „Staatsgrundgesetz über die allgemeinen Rechte der Staatsbürger" aus 1867 als (klassisch-liberalen) Grundrechtekatalog im Verfassungsrang. In Weimar hingegen verabschiedete man einen neuen Katalog, welcher auch soziale Grundrechte beinhaltete. Während die Fassung des B-VG aus 1920 eine rein parlamentarische Regierungsform beinhaltete, wurde 1929 auch die österreichische Regierungsform stark weimarisiert, indem eine halbparlamentarische Regierungsform eingeführt wurde. ${ }^{7}$ Diese Novelle hatte also eine Stärkung der Rolle des Bundespräsidenten und dem gleichzeitig eine Schwächung der Rolle des Nationalrates $(z B$ durch die Einschränkung der Immunität der Abgeordneten zum Nationalrat) zur Folge. ${ }^{8}$ Dies werden wir sogleich im nächsten Punkt sehen, indem wir feststellen, dass zu diesem Zweck zahlreiche konkrete Bestimmungen im B-VG abgeändert wurden. ${ }^{9}$

\section{DIE PARTIELLE WEIMARISIERUNG DES B-VG IN 1929}

Die WRV entfaltete eben nicht nur bei der Verabschiedung des B-VG 1920 einen Einfluss auf das B-VG, sondern auch bei der großen Verfassungsreform 1929, deren Hauptgrund gerade eine weitere Angleichung an die WRV und somit eine Vorbereitung des Anschlusses war. ${ }^{10}$ Neben diesem primären Bewegungsgrund für die Novelle 1929 gab es auch inhaltliche Gründe, die aber teilweise wieder durch Argumente inspiriert waren, die in Weimar geboren wurden. Das betraf vor allem die Übermacht des Parlaments, welcher ein erwünschtes "Gegengewicht“ durch den Präsidenten im Sinne einer Gewaltenteilung entgegen gestellt wurde. ${ }^{11}$ Teilweise stellten diese Argumente aber auch eine Nachahmung des monarchischen Prinzips dar. ${ }^{12}$

6 Hans Kelsen, Österreichisches Staatsrecht, 1923, p. 238. Für die Unabhängigkeit standen vor allem katholisch-konservative (vormals monarchische) Kräfte.

7 Anton Pelinka spricht von einer „antiparlamentarischen Zeitgeistigkeit“ bezüglich der 1929er Novelle, siehe Anton Pelinka, „Nicht schon wieder Weimar. Zur Sehnsucht nach einem starken Bundespräsidenten“, Journal für Rechtspolitik 1994, p. 140.

8 Wilhelm Brauneder, Österreichische Verfassungsgeschichte, Manz, Wien, 8. Aufl. 2001, p. 215.

9 Hans Kelsen, „Die Verfassungsreform“, Juristische Blätter 1929, pp. 445 et seq.; Hans Kelsen, „Die Verfassung Österreichs“, Jahrbuch des öffentlichen Rechts 1930, pp. 130 et seq.; Adolf Merkl, „Zur Verfassungsreform. Die Verfassungsnovelle im Lichte der Demokratie“, Juristische Blätter 1929, pp. 469 et seq.; Adolf Merkl, „Der rechtliche Gehalt der österreichischen Verfassungsreform vom 7. Dezember 1929“, Zeitschrift für öffentliches Recht 1931, pp. 161 et seq.

10 Klaus Berchtold, Die Verfassungsreform von 1929, Braumüller, Wien, 1979.

11 So gehen zB die Stimmen gegen den Parlamentsabsolutismus auf Hugo Preuß zurück, nach dem die Direktwahl des Reichspräsidenten ihm die Möglichkeit gibt, „dem Kleinkriege der Parteien und ihren Eintagsintrigen ein Gegengewicht zu bieten, ihnen gegenüber den großen Grundfragen des politischen Lebens zur Geltung zu bringen. Würde der Präsident statt unmittelbar durch das Volk vom Reichstage gewählt, so ergäbe sich statt der parlamentarischen Demokratie ein 
In der ursprünglichen 1920er Fassung des B-VG konnte nur der Nationalrat sich selbst durch Gesetz auflösen. Nach der BVG-Novelle-1929 konnte allerdings auch der Bundespräsident den Nationalrat auflösen (inspiriert durch die ähnliche Kompetenz des Reichspräsidenten gemäß Art 25 WRV). ${ }^{13}$

Gemäß der ursprünglichen 1920er Fassung des B-VG wurde der Bundespräsident durch die Bundesversammlung mit einmaliger Wiederwählbarkeit für 4 Jahre gewählt (Art 60 Abs 1 B-VG). Mit der 1929er Novelle wurde aber die Direktwahl eingeführt und das Mandat auf 6 Jahre verlängert. Diese Änderung war auch durch die Regelung des Art 41 WRV inspiriert, nach der der Reichspräsident direkt vom Reichsvolk auf 7 Jahre gewählt wurde. ${ }^{14}$

Gemäß der ursprünglichen 1920er Fassung des B-VG konnte nur der Nationalrat die Regierung entlassen (und der Bundeskanzler wurde nur vom Nationalrat gewählt). Mit der Novelle 1929 wurde - inspiriert durch die Art 53-54 WRV - die Kompetenz des Bundespräsidenten etabliert, den Kanzler zu entlassen. Somit wurde eine doppelte Verantwortlichkeit eingeführt, da die Regierung auch weiterhin durch ein Misstrauensvotum gestürzt werden konnte. Der Oberbefehl der Armee lag 1920 beim Nationalrat, seit 1929 liegt dieser nach Weimarer Muster beim österreichischen Bundespräsidenten (allerdings mit Hinweis der Einhaltung der Gesetze). In der 1920er Textversion des B-VG haben die Notstandskompetenzen völlig gefehlt (bis auf die Sitzverlegung des Nationalrates für die Dauer außerordentlicher Verhältnisse gemäß Art 25 Abs 2 B-VG). ${ }^{15}$ Eine dem Art 48 WRV ähnliche Regelung wurde letztendlich in Österreich 1929 nicht eingeführt, aber eine abgeschwächte Version des Notverordnungsrechts (insb mit Vorschlagsrecht und Gegenzeichnung der Bundesregierung gemäß den neuen Art 18 Abs 3-5 B-VG) schon.

Die 1920 noch fehlende Möglichkeit der Absetzung des Bundespräsidenten durch eine Volksabstimmung wurde 1929 (nach dem Muster von Art 43 Abs 2 WRV, stellenweise sogar wortwörtlich übernommen) ${ }^{16}$ auch eingeführt (Art 60 Abs 6 B-VG):

schrankenloser Parlaments-Absolutismus." Hugo Preuß, Gesammelte Schriften. Band 4, Mohr Siebeck, Tübingen, 2008, p. 109. Zum Demokratiekonzept der WRV („Parlamentarismus mit Gegengewichten“) siehe Christoph Gusy, 100 Jahre Weimarer Verfassung. Eine gute Verfassung in schlechter Zeit, Mohr Siebeck, Tübingen, 2018, pp. 118 et seq. Zu den damaligen österreichischen rechtswissenschaftlichen Debatten, auch mit zahlreichen Hinweisen auf Weimar, siehe Gernot D Hasiba, Die zweite Bundes-Verfassungsnovelle von 1929, Böhlau, Wien, 1976, pp. 78 et seq. 58.

12 Kurt Strele, Rechtsstaat und Demokratie im neuen Österreich, Wagner, Innsbruck, 1931, p.

13 Sima, op. cit., p. 139.

14 Sima, op. cit., p. 193.

15 Bernd Wieser, „Art 25 B-VG“, in: Karl Korinek et al (Hrsg), Bundesverfassungsrecht, Verlag Österreich, Wien, 1. Lfg. 1999, Rz 1.

16 Art 43 Abs 2 WRV: „Vor Ablauf der Frist kann der Reichspräsident auf Antrag des Reichstags durch Volksabstimmung abgesetzt werden. Der Beschluß des Reichstags erfordert Zweidrittelmehrheit. Durch den Beschluß ist der Reichspräsident an der ferneren Ausübung des Amtes verhindert. Die Ablehnung der Absetzung durch die Volksabstimmung gilt als neue Wahl und hat die Auflösung des Reichstags zur Folge." 


\begin{abstract}
„Vor Ablauf der Funktionsperiode kann der Bundespräsident durch Volksabstimmung abgesetzt werden. Die Volksabstimmung ist durchzuführen, wenn die Bundesversammlung es verlangt. Die Bundesversammlung ist zu diesem Zweck vom Bundeskanzler einzuberufen, wenn der Nationalrat einen solchen Antrag beschlossen hat. Zum Beschluss des Nationalrates ist die Anwesenheit von mindestens der Hälfte der Mitglieder und eine Mehrheit von zwei Dritteln der abgegebenen Stimmen erforderlich. Durch einen derartigen Beschluss des Nationalrates ist der Bundespräsident an der ferneren Ausübung seines Amtes verhindert. Die Ablehnung der Absetzung durch die Volksabstimmung gilt als neue Wahl und hat die Auflösung des Nationalrates (Art. 29 Abs. 1) zur Folge. Auch in diesem Fall darf die gesamte Funktionsperiode des Bundespräsidenten nicht mehr als zwölf Jahre dauern."
\end{abstract}

Die Regierungsform Österreichs war bis 1929 eindeutig parlamentarisch. Seit der Novelle 1929 ist die Regierungsform gemäß der Inspiration durch die WRV semi-präsidentiell, ${ }^{17}$ obwohl in der politischen Praxis die präsidentiellen Kompetenzen nicht ausgeübt werden („Rollenverzicht“). ${ }^{18}$ Die Wirkung der WRV hat dazu geführt, dass statt der bis dahin rechtlich klar abgegrenzten Kompetenzen „Führungskonkurrenzen von Kanzler, Vizekanzler und Bundespräsident [...] im B-VG angelegt“ wurden. ${ }^{19}$

In der konstitutionellen Monarchie war die Gewalt des Staatsoberhauptes die letzte Garantie gegen die Volkssouveränität. ${ }^{20}$ Das Weimarer Modell, das 1929 auch in Österreich übernommen wurde, ist eine Nachahmung des monarchischen Prinzips, und somit eher eine Schwächung, als eine Stärkung der Volkssouveränität. Hans Kelsen schrieb schon 1919 über das Verhältnis zwischen Exekutive und Legislative treffend wie folgt (die Frage der Justiz wurde hier ausgeklammert): ${ }^{21}$

\begin{abstract}
„der politische Gedanke der Volkssouveränität, sofern er in der repräsentativen Demokratie verwirklicht ist, [ist] mit einer Loslösung der vollziehenden Gewalt von dem Gesetzgebungsorgan des Parlaments schwer vereinbar [...]. Sicherlich wäre es denkbar, daß durch unmittelbare Volkswahlen zwei voneinander verschiedene Organe für Gesetzgebung und Verwaltung geschaffen werden. In der Präsidentschaftsrepublik, in der das Staatsoberhaupt ebenso wie der Gesetzgebungskörper vom Volke gewählt wird, ist dieser Gedanke verwirklicht. Indes tritt gerade in der Präsidentschaftsrepublik mit weitgehender Trennung der Gewalten - wie etwa in den Vereinigten Staaten von Amerika der mit der Gewaltentrennung verbundene Gegensatz zum Prinzip der Volkssouveränität deutlich hervor. Diese Gewaltentrennung hat, wenn nicht schon die Absicht so doch die
\end{abstract}

17 Wolfgang C Müller, „Austria: The 'Authority in Reserve' Presidency“, in: Robert Elgie (Hrsg), Semi-Presidentialism in Europe, Oxford University Press, Oxford, 1999, pp. 22 et seq.

18 David Wineroither, „Bundespräsident und Bundeskanzler: Konsens, Konflikt oder Neutralität?", Österreichisches Jahrbuch für Politik 2006, pp. 603 et seq.

19 Manfried Welan, „Präsidialismus oder Parlamentarismus? Demokratiepolitische Perspektiven“, in: David F. J. Campbell et al. (Hrsg.), Die Qualität der österreichischen Demokratie. Versuche einer Annäherung, Opladen, Wien, 1996, pp. 59 et seq., 69.

20 Sima op. cit., p. 228.

21 Hans Kelsen, „Die Organisation der vollziehenden Gewalt Deutschösterreichs nach der Gesetzgebung der konstituierenden Nationalversammlung", Zeitschrift für öffentliches Recht 1919, p. 53. Etwas später bemerkt er (etwas kontrovers) noch (ibid. p. 54): „Je größer das Parlament, desto leichter kann es zu einem Abbild der politischen Strebungen im Volke gestaltet, desto leichter kann es aber auch als ein solches Spiegelbild der Volksmeinung erhalten werden." 
Folge, daß das Gesetzgebungsorgan, in dem das Volk wegen der Vielköpfigkeit dieses Organs, wenn überhaupt so nur hier repräsentiert wird, keineswegs als das höchste sich geltend machen kann. Ist die vollziehende Gewalt einem Monarchen übertragen, dann tritt dieser als gleichgeordnete, ja womöglich sogar als übergeordnete Macht der an der Gesetzgebung mitbeteiligten Volksvertretung entgegen. Das politische Axiom der Trennung der Gewalten ist nicht zuletzt zu dem Zwecke geschaffen worden, um dem aus dem Absolutismus in die Beschränkung des Konstitutionalismus verdrängten Monarchen ein letztes Übergewicht über die im Parlament konzentrierte Gewalt des Volkes zu sichern. Es ist zweifellos nur die bewußte oder unbewußte Nachahmung des monarchischen Prinzips, wenn in der sogenannten Präsidentschaftsrepublik die vollziehende Gewalt auf einem Präsidenten übertragen wird, der nicht aus der Volksvertretung hervorgeht, sondern unmittelbar durch das Volk gewählt wird und wenn auch in anderer Weise die Unabhängigkeit des mit der vollziehenden Gewalt betrauten Präsidenten gegenüber der Volksvertretung gesichert wird. So paradox es zunächst erscheinen mag, so bedeutet doch die unmittelbare Wahl des Präsidenten durch das Volk eher eine Schwächung als eine Stärkung des Prinzips der Volkssouveränität.“

\section{UNTERSCHIEDE ZWISCHEN DEM B-VG NACH 1929 UND DER WRV}

Der wahrscheinlich wichtigste Unterschied zwischen der WRV und dem BVG bestand in der Demokratieauffassung. Eben dieser Unterschied wurde aber durch die B-VG Novelle 1929 aufgegeben. Daneben gab es noch Unterschiede, die eher mit den Umständen bei der Verabschiedung zu erklären sind. So wurde auf Grund ideologischer Kontroversen auf eine Präambel verzichtet und - wie bereits erwähnt - kein neuer Grundrechtekatalog geschaffen, sondern auf die Grundrechte des StGG 1867 verwiesen.

Es gab aber auch einige Unterschiede zwischen dem B-VG nach 1929 und der WRV, welche die Weimarisierung von 1929 überlebt haben. Das sind Unterschiede, die bewusst beibehalten wurden und meistens zumindest teilweise mit theoretischen Positionen von Hans Kelsen koinzidieren. ${ }^{22}$

Im österreichischen Verfassungstext findet sich auch nach 1929 nicht die Formulierung des Art 1 WRV, nach welcher es heißt: „Die Staatsgewalt geht vom Volke aus“. Das B-VG beinhaltet dieselbe Bestimmung, allerdings ohne die traditionelle Gewaltenteilungsterminologie: Das „Recht [der Republik] geht vom Volke aus“ (Art 1 B-VG). Dies ist darauf zurückzuführen, dass für Kelsen der Staat mit der Rechtsordnung identisch war. Demzufolge könne es keine Gewalt außerhalb des Rechts geben.23 So war und ist der österreichische Verfassungstext im Gegensatz zur WRV und zum Grundgesetz „gewaltfrei“.24

Die WRV beruht grundsätzlich auf der Grundlage der Dreiteilung Montesquieus. ${ }^{25}$ Im System des B-VG heißt es aber „Drittes Hauptstück.

22 András Jakab, „Die österreichische öffentlichrechtliche Dogmatik aus deutschem Blickwinkel. Ex contrario fiat lux", Der Staat 2007, pp. 268 et seq.

${ }^{23}$ Hans Kelsen, Hauptprobleme der Staatsrechtslehre, entwickelt aus der Lehre vom Rechtssatze, Mohr, Tübingen, 1911. Mehr dazu bei Theo Öhlinger, Hans Kelsen und das österreichische Bundesverfassungsrecht [Vortrag im Rahmen der Tagung „Die österreichischen Einflüsse auf die Modernisierung des japanischen Rechts“, Wien, 20.3.2003], www.univie.ac.at/staatsrechtoehlinger $/ \mathrm{php} /$ get.php?id=143, p. 2.

${ }^{24}$ Ewald Wiederin, „Österreich“, in: Armin von Bogdandy/Pedro Cruz Villalón/Peter M. Huber (Hrsg.), Ius Publicum Europaeum, Band I, Müller, Heidelberg, 2006, pp. 389 et seq.

$25 \mathrm{Ob}$ der Reichspräsident der WRV als eine vierte Gewalt, eine pouvoir neutre (im Sinne von Benjamin Constant) verstanden werden kann, soll hier offen bleiben. Zu dieser Kontroverse siehe 
Vollziehung des Bundes“, und darunter „A. Verwaltung“ bzw „B. Ordentliche Gerichtsbarkeit“. Das heißt, der Oberbegriff „Vollziehung“ beinhaltet sowohl die Verwaltung, als auch die ordentliche Gerichtsbarkeit. Der Grund dafür liegt in einer neuen Gewaltenteilungslehre, welche die Konsequenz des reinen Parlamentarismus ist. Gemäß der Stufenbaulehre sind der die Demokratie ausdrückende Gesetzgebung die beiden anderen Gewalten (Exekutive und Judikative, zwar voneinander getrennt aber) ${ }^{26}$ gleichermaßen untergeordnet, da die Rechtsakte der Exekutive und der Judikative ihre Geltung aus den Gesetzen beziehen. Dies bedeutet letztlich, dass es nur zwei Grundfunktionen (Gewalten) gibt, nämlich die Gesetzgebung und die Vollziehung. Innerhalb der Vollziehung agieren die Verwaltung und die Judikative jedoch voneinander getrennt. ${ }^{27}$ Dies bedeutet eine betont dominante Rolle des Parlaments gegenüber den zwei anderen Gewalten (Stufenbau der Staatsfunktionen). ${ }^{28}$

Die klare und detaillierte Ausgestaltung der Verfassungsgerichtsbarkeit im B-VG (zumeist direkt aus der Feder von Hans Kelsen), inklusive der Überprüfung von Gesetzen (Art 140 B-VG), fehlt in der WRV. Die Anordnung, dass Verfassungsgesetze als solche explizit bezeichnet werden müssen (Art 44 Abs 1 BVG) gab es in der WRV ebenso wenig (Art 76 WRV). Die Mehrschichtigkeit der Verfassung gemäß Art 44 Abs 2 B-VG (durch Volksabstimmung erschwertes Verfahren für besonders schwerwiegende Verfassungsänderungen, heute Art 44 Abs 3 B-VG) war in Weimar ebenso unbekannt.

In Österreich gab es außerdem nie eine dem Art 13 Abs 1 WRV („Reichsrecht bricht Landesrecht. ") ähnliche Bestimmung: mit einigen Ausnahmen wurden alle Kompetenzen durch den Bundesverfassungsgesetzgeber entweder als Bundesoder als Landeskompetenzen (sog. Prinzip der Exklusivität). Das B-VG hat ferner die sogenannte mittelbare Bundesverwaltung als Grundregel eingeführt: Der Bund darf grundsätzlich keine eigene Verwaltung in den Ländern haben, es sei denn das B-VG erlaubt es explizit (Art 102 B-VG). Gemäß Art 14 WRV hingegen kann als Hauptregel die Bundesverwaltung durch einfaches Reichsgesetz eingeführt werden. Die Regelung des B-VG bedeutete eine wesentlich stärkere Stellung der Länder, was juristisch (wenn auch weniger faktisch) als ein Schutz gegen Zentralisierungstendenzen angesehen werden kann.

\section{ZWEI UNTERGANGSGESCHICHTEN}

Als ein symbolischer Akt des Unterganges der Ersten Österreichischen Republik kann der Brand des Wiener Justizpalastes 1927 betrachtet werden was aber im Gegensatz zum Reichstagsbrand 1933 kein Anlass zur Einführung der Diktatur war, sondern eher nur ein Symptom der demokratischen Erosion. In einer politisch-ideologisch stark polarisierten Umgebung wurden drei Mitglieder eines Wehrverbandes freigesprochen, die in Burgenland während einem Zusammenstoß mit Sozialdemokraten zwei Menschen erschossen haben (sog.

Carl Schmitt, Der Hüter der Verfassung, Mohr, Tübingen, 1931, und Hans Kelsen, Wer soll der Hüter der Verfassung sein?, Rothschild, Berlin, 1931.

${ }^{26}$ Art 94 Abs 1 B-VG: „Die Justiz ist von der Verwaltung in allen Instanzen getrennt.“

27 Theo Öhlinger, Der Stufenbau der Rechtsordnung, Manz, Wien, 1975, p. 29.

28 Öhlinger, Der Stufenbau der Rechtsordnung, op. cit., p. 30. 
Schattendorfer Urteil). Darauf brachen gewaltsame Demonstrationen gegen das gerichtliche Urteil in Wien aus, während dessen auch der Wiener Justizpalast niedergebrannt wurde. ${ }^{29}$

Die Gesetzgebung der Ersten Republik in Österreich wurde mithilfe einer Kombination aus Rechtslücke und illegaler Gewaltanwendung 1933 ausgeschaltet (sog. Selbstausschaltung des Nationalrates). ${ }^{30}$ Wegen der knappen Mehrheitsverhältnisse im Nationalrat haben auf einmal alle drei Präsidenten des Nationalrates ihr Amt niedergelegt (damit sie an der Stimmabgabe teilnehmen können). Darauf hat die Bundesregierung die Selbstausschaltung des Nationalrates verkündet und gleichzeitig eine Neuberufung mit Sicherheitsbeamten verhindert.

Obwohl im B-VG kein dem Art 48 WRV ähnliches Notverordnungsrecht bis 1929 existierte, wurde das sog. Kriegswirtschaftliche Ermächtigungsgesetz (KWEG, Gesetz vom 24.7.1917, RGB1 307), das aus dem Ersten Weltkrieg stammte, in der Verfassungspraxis nach der Selbstausschaltung des Nationalrates für ähnliche Zwecke benutzt. Dieses Gesetz ermächtigte die Regierung, „während der Dauer der durch den Krieg hervorgerufenen außerordentlichen Verhältnisse durch Verordnung die notwendigen Verfügungen zur Förderung und Wiederaufrichtung des wirtschaftlichen Lebens, zur Abwehr wirtschaftlicher Schädigungen und zur Versorgung der Bevölkerung mit Nahrungsmitteln und anderen Bedarfsgegenständen zu treffen." Solche Verordnungen hätten also zumindest mittelbar wirtschaftlich bedingt und „durch den Krieg hervorgerufen" sein müssen. Auf Grund einer sehr weiten Auslegung wurde das Gesetz allerdings auch 1933 zur Anwendung gebracht. ${ }^{31}$ Mit solchen KWEG-Verordnungen der Dolfuß-Regierung wurden 1933 die Rede-, Presse- und Versammlungsfreiheit stark eingeschränkt, ${ }^{32}$ das Verfassungsgerichtshofgesetz abgeändert (und somit der Verfassungsgerichtshof praktisch lahmgelegt). ${ }^{33}$ Ein Jahr später wurde 1934 sogar die neue ständisch-autoritäre Verfassung (!) auf dieser Rechtsgrundlage verabschiedet. ${ }^{34}$

Sowohl die Weimarer Republik, als auch die Erste Österreichische Republik sind nach ca. anderthalb Jahrzehnten ungefähr zeitglich 1933 untergegangen. In

29 Walter Goldinger, „Der geschichtliche Ablauf der Ereignisse in Österreich 1918-1945“, in: Heinrich Benedikt (Hrsg), Geschichte der Republik Österreich, Verlag für Politik und Geschichte, Wien, 1954, pp. 147 et seq.; Oskar Lehner, Österreichische Verfassungs- und Verwaltungsgeschichte. Mit Grundzügen der Wirtschafts- und Sozialgeschichte, Trauner Verlag, Linz, 4. Aufl. 2007, p. 287; Walter B Simon, Österreich 1918-1938, Böhlau, Wien, 1984, pp. 106 et seq.

30 Susanne Janistyn-Novák, „Vorwort“, in: Parlamentsdirektion (Hrsg), Staats- und Verfassungskrise 1933, Böhlau, Wien, 2014, pp. 7 et seq.

${ }^{31}$ Simon, op. cit., p. 147; Brauneder, op. cit., p. 234.

32 Simon, op. cit., p. 153.

33 Brauneder, op. cit., p. 233; Klaus Berchtold, Verfassungsgeschichte der Republik Österreich. Band 1: 1918-1933. Fünfzehn Jahre Verfassungskampf, Springer, Wien, 1998, p. 752.

34 Alfred J Noll / Emmerich Tálos, „Streit um Worte oder Streit um die Sache? Zu Ludwig Adamovich" „Das autoritäre System 1933 - 1938 zwischen Rechtsbruch und Gewissensentscheidung “", Journal für Rechtspolitik 2015, pp. 277 et seq., 278. Kurz darauf wurde die 1934 Verfassung noch einmal (!), diesmal im Nationalrat, mit schwerwiegenden Verfahrensbrüchen, also schon wieder illegal verabschiedet. 
beiden Ländern wurde der Untergang sowohl damals, als auch heute als illegal (also als Verfassungsbruch) betrachtet, was aber am faktischen Resultat nichts änderte. ${ }^{35}$ In Österreich wurde 1934 ein autoritäres-ständestaatliches Regime eingeführt, was als eine betont katholische ${ }^{36}$ und autoritär-konservative österreichische Alternative anstatt des Anschlusses an das nationalsozialistische Deutschland gesehen wurde. ${ }^{37} 1938$ wurden letztendlich die zwei Staaten mit dem Anschluss Österreichs an Deutschland vereint. 38

Als Gründe für den Untergang der Ersten Österreichischen Republik werden üblicherweise die Wirtschaftskrise, ${ }^{39}$ die international forcierte soziale Sparmaßnahmen (Anleihe vom Völkerbund durch das sog. „Lausanner Protokoll“, 1932 (BGBl 1933/12)), ${ }^{40}$ die fehlende demokratische Kultur, ${ }^{41}$ und die Polarisierung und Militarisierung des politischen Lebens (was 1934 praktisch zum Bürgerkrieg geführt hat) genannt. ${ }^{42}$ All diese Phänomene waren in ähnlicher Weise auch in der Weimarer Republik gegeben.

\section{ERBE}

Die WRV wurde formal am Anfang des Nationalsozialismus nicht außer Kraft gesetzt; das B-VG wurde hingegen formal mit der ständestaatlichen Verfassung 1934 (Maiverfassung, in welcher auch der offizielle Name „Republik Österreich“ auf „Bundesstaat Österreich“ ausgetauscht wurde) ${ }^{43}$ außer Kraft gesetzt. ${ }^{44}$ Nach

35 Gerhard G Senft, Im Vorfeld der Katastrophe. Die Wirtschaftspolitik des Ständestaates. Österreich 1934-1938, Braumüller, Wien, 2002, p. 18; Rudolf Machacek, „80 Jahre B-VG - 80 Jahre VfGH. 150 Jahre RA-Kammer für Wien und NÖ. Aus Anlass des 60. Geburtstages von Univ.-Prof. Dr. Karl Korinek, Vize-Präsident des VfGH," Anwaltsblatt 2000, pp. 652 et seq.; Theo Öhlinger / Harald Eberhard, Verfassungsrecht, facultas, Wien, 11. Aufl. 2016, p. 46.

36 Zur Katholischen Kirche als „Herrschaftsträger und Ideologielieferant“ siehe Ernst Hanisch, „Der Politische Katholizismus als ideologischer Träger des „Austrofaschismus“", in: Emmerich Tálos / Wolfgang Neugebauer (Hrsg): „Austrofaschismus“. Beiträge über Politik, Ökonomie und Kultur 1934-1938, Verlag für Gesellschaftskritik, Wien, 3. Aufl. 1984, p. 53.

37 Ludwig Adamovich, „Das autoritäre System 1933-1938 zwischen Rechtsbruch und Gewissensentscheidung“, Journal für Rechtspolitik 2015, p. 126.

38 Ulrich Kluge, Der österreichische Ständestaat 1934-1938, Verlag für Geschichte und Politik, Wien, 1984; Wolfgang Putschek, Ständische Verfassung und autoritäre Verfassungspraxis in Österreich 1933-1938, Peter Land, Frankfurt am Main, 1993; Ewald Wiederin, "Christliche Bundesstaatlichkeit auf ständischer Grundlage: Eine Strukturanalyse der Verfassung 1934“, in: Reiter-Zatloukal ea (Hrsg), Österreich 1933-1938, Böhlau, Wien, 2012, p. 31.

39 Hans Kernbauer / Eduard März / Fritz Weber, „Die wirtschaftliche Entwicklung“, in: Erika Weinzierl / Kurt Skalnik (Hrsg), Österreich 1918-1938. Geschichte der Ersten Republik, Band 1, Styria, Graz, 1983, pp. 343 et seq.; Senft, op. cit., p. 47; Goldinger, op. cit., p. 191.

40 Lehner, op. cit., p. 282; Senft, op. cit., p. 37; Berchtold, Verfassungsgeschichte, op. cit., pp. 350 et seq.

41 Adamovich, „Das autoritäre System 1933-1938“op. cit., p. 126.

42 Ludwig Adamovich / Bernd-Christian Funk / Gerhart Holzinger / Stefan Leo Frank, Österreichisches Staatsrecht, Verlag Österreich, Wien, 2. Aufl. 2011, p. 80.

43 Heinz Fischer, „Festvortrag „100 Jahre Republik - 100 Jahre Justizgeschichte“ Richtertag 2017“, Österreichische Richterzeitung 2018, p. 24. 
der Befreiung von dem nationalsozialistischen Unrechtsregime hatte die WRV eine starke Wirkung auf das deutsche Grundgesetz (sowohl im negativen, als auch im positiven Sinne). Das B-VG wurde nach dem Zweiten Weltkrieg formal wieder in Kraft gesetzt. In der ersten Nummer des neu erschienenen „Staatsgesetzblatt für die Republik Österreich“ (1. Mai 1945) wurde die Unabhängigkeitserklärung verkündet, die (bis heute) als Grundlage der österreichischen Rechtsordnung nach 1945 gilt. Die Präambel beinhaltete die österreichische Opferthese (nach der Österreich nur ein Opfer des deutschen Nationalsozialismus gewesen sei, und keine Mitschuld im Zweiten Weltkrieg trage). Die Erklärung beinhaltete unter anderem auch die folgenden Bestimmungen:

Art. I: Die demokratische Republik Österreich ist wiederhergestellt und im Geiste der Verfassung von 1920 einzurichten.

Art. II: Der im Jahre 1938 dem österreichischen Volke aufgezwungene Anschluß ist null und nichtig.

In Österreich wurde also nach dem Zweiten Weltkrieg keine neue Verfassung erlassen, sondern einfach das B-VG wieder in Kraft gesetzt (was bis heute die Verfassung Österreichs ist). Dies unter anderem deshalb, „weil auf deren Inhalt sowohl die Alliierten, als auch die Kommunisten hätten einwirken können. Außerhalb war sie deshalb stringent, weil es unmittelbar nach dem zweiten verlorenen Krieg neben dem B-VG 1920 wenig gab, was eine demokratische österreichische Identität hätte stiften können“. 45 Als Datum der Wiedereinsetzung wurde gemäß Art 1 Verfassungs-Überleitungsgesetz 1945 für das B-VG das Jahr 1929 (also nach der partiellen Weimarisierung) gewählt. ${ }^{46}$ So lebt auch die Wirkung der WRV bis heute in einigen Bestimmungen des B-VG weiter. Die 1929 eingeführten Weimarer Vulnerabilitäten sind bis heute Grund für Besorgnis in der österreichischen Verfassungsrechtswissenschaft. ${ }^{47}$ Bisher hat dies nur deshalb zu keinen größeren verfassungspolitischen Problemen geführt, weil die

44 Derogiert durch Art 181 Verfassung 1934, bzw § 1 und § 56 Bundesverfassungsgesetz vom 19. Juni 1934, betreffend den Übergang zur ständischen Verfassung (Verfassungsübergangsgesetz 1934).

45 Ewald Wiederin, „Denken vom Recht her. Über den modus austriacus in der Staatsrechtslehre“, in: Helmuth Schulze-Fielitz (Hrsg.), Staatsrechtslehre als Wissenschaft, Duncker \& Humblot, Berlin, 2007, pp. 293, 312.

46 Für einfache Gesetze war es 1933, siehe Art 1 Verfassungs-Überleitungsgesetz (V-ÜG, Langtitel: Verfassungsgesetz über das neuerliche Wirksamwerden des BundesVerfassungsgesetzes in der Fassung von 1929) (StGB1. Nr. 4/1945, p. 7) vom 13. Mai rückdatiert auf den 1. Mai 1945. Ein früheres Datum hätte einerseits Mitschuld für solche Politiker impliziert, die man nach dem Zweiten Weltkrieg für den Wiederaufbau brauchte. Andererseits hätte es auch praktisch-rechtstechnisch zu Komplikationen geführt, die man lieber vermeiden wollte. Siehe zB Ludwig Adamovich, [ohne Titel] in: Nikolaus Grass (Hrsg), Österreichische Rechts- und Staatswissenschaften der Gegenwart in Selbstdarstellungen, Wagner, Innsbruck, 1952, p. 18; Ewald Wiederin, „Einheitsstaat oder Bundesstaat? Diskurse und Entscheidungen 1945“, in: Martin P Schennah (Hrsg), Rechtshistorische Aspekte des Föderalismus, Verlag Österreich, Wien, 2015, p. 189.

47 Theo Öhlinger / Maximilian Steinbeis, „Das wäre wohl so etwas wie eine Verfassungskrise“ [Interview], VerfBlog, 2016/4/25, https://verfassungsblog.de/das-waere-wohl-so-etwas-wie-eineverfassungskrise/. 
jeweiligen Bundespräsidenten in der Praxis self-restraint ausgeübt haben. Die formal-rechtliche Konstruktion für die Ausübung einer das Parlament sehr stark einschränkende präsidentiellen Rolle ist aber seit 1929 da, ${ }^{48}$ und es gibt keine Garantie dafür, dass dieser Rollenverzicht für immer ausgeübt werden wird. ${ }^{49}$ Weimar wirft also immer noch einen bedrohlichen Schatten auf das österreichische Verfassungssystem.

\section{LITERATUR}

Ludwig Adamovich, in: Nikolaus Grass (Hrsg), Österreichische Rechts- und Staatswissenschaften der Gegenwart in Selbstdarstellungen, Wagner, Innsbruck, 1952, pp. 10 et seq.

Ludwig Adamovich, „Das autoritäre System 1933-1938 zwischen Rechtsbruch und Gewissensentscheidung“, Journal für Rechtspolitik 2015, pp. 126 et seq.

Ludwig Adamovich / Bernd-Christian Funk / Gerhart Holzinger / Stefan Leo Frank, Österreichisches Staatsrecht, Verlag Österreich, Wien, 2. Aufl. 2011, p. 80.

Klaus Berchtold, Die Verfassungsreform von 1929, Braumüller, Wien, 1979.

Klaus Berchtold, Verfassungsgeschichte der Republik Österreich. Band 1: 19181933. Fünfzehn Jahre Verfassungskampf, Springer, Wien, 1998.

Wilhelm Brauneder, Österreichische Verfassungsgeschichte, Manz, Wien, 8. Aufl. 2001.

Felix Ermacora, Die Entstehung der Bundesverfassung 1920. Band II, Braumüller, Wien, 1989.

Heinz Fischer, „Festvortrag „100 Jahre Republik - 100 Jahre Justizgeschichte“ Richtertag 2017“, Österreichische Richterzeitung 2018, pp. 22 et seq.

Walter Goldinger, „Der geschichtliche Ablauf der Ereignisse in Österreich 19181945“, in: Heinrich Benedikt (Hrsg), Geschichte der Republik Österreich, Verlag für Politik und Geschichte, Wien, 1954, pp. 147 et seq.

Christoph Gusy, 100 Jahre Weimarer Verfassung. Eine gute Verfassung in schlechter Zeit, Mohr Siebeck, Tübingen, 2018.

48 Friedrich Koja, „Die rechtlichen und politischen Möglichkeiten des Bundespräsidenten bei der Ernennung der Bundesregierung“, Journal für Rechtspolitik 1994, pp. 175 et seq.

49 Anton Pelinka, op. cit., p. 181. Die Frage ist nicht nur von rein akademischem Interesse. Während der letzten Präsidentschaftswahlen 2016 hat der Kandidat Norbert Hofer (FPÖ), der im dritten Wahlgang 46\% der Stimmen bekommen und somit die Wahl nur knapp verloren hat, bekanntlich dafür argumentiert, dass der Bundespräsident seine formellen Kompetenzen viel aktiver ausüben sollte als dies bisher der Fall war. Er hatte zum Beispiel angekündigt seine formelle Rolle beim Erlass von Gesetzen und der Ratifikation von Staatsverträgen seine Unterschrift zu verweigern. Außerdem hat er während der Kampagne im Österreichischen Rundfunk live bezüglich der Kompetenzen des Bundespräsidenten gesagt: „Sie werden sich noch wundern, was alles gehen wird.“ (21. April 2016, ORF Sendung „Elefantenrunde“, siehe https://wien.orf.at/news/stories/2773586/). Zuletzt zu diesen Fragen optimistischer, dennoch mit einigen Änderungsvorschlägen zum B-VG, siehe Benjamin Kneihs, „Die „demokratische Republik“: Zu den Befugnissen des Bundespräsidenten“, Journal für Rechtspolitik 2017, pp. 73 et seq. 
Ernst Hanisch, „Der Politische Katholizismus als ideologischer Träger des „Austrofaschismus“", in: Emmerich Tálos / Wolfgang Neugebauer (Hrsg): „Austrofaschismus“. Beiträge über Politik, Ökonomie und Kultur 1934-1938, Verlag für Gesellschaftskritik, Wien, 3. Aufl. 1984, pp. 53 et seq.

Gernot D Hasiba, Die zweite Bundes-Verfassungsnovelle von 1929, Böhlau, Wien, 1976.

Susanne Janistyn-Novák, „Vorwort“, in: Parlamentsdirektion (Hrsg), Staats- und Verfassungskrise 1933, Böhlau, Wien, 2014, pp. 7 et seq.

András Jakab, „Die österreichische öffentlichrechtliche Dogmatik aus deutschem Blickwinkel. Ex contrario fiat lux”, Der Staat 2007, pp. 268 et seq.

Hans Kelsen, Hauptprobleme der Staatsrechtslehre, entwickelt aus der Lehre vom Rechtssatze, Mohr, Tübingen, 1911.

Hans Kelsen, „Die Organisation der vollziehenden Gewalt Deutschösterreichs nach der Gesetzgebung der konstituierenden Nationalversammlung“, Zeitschrift für öffentliches Recht 1919, pp. 48 et seq.

Hans Kelsen / Adolf Merkl / Georg Froehlich, Die Bundesverfassung vom 1. Oktober 1920, Deuticke, Wien, 1922

Hans Kelsen, „Die Verfassungsreform“, Juristische Blätter 1929, pp. 445 et seq.

Hans Kelsen, „Die Verfassung Österreichs“, Jahrbuch des öffentlichen Rechts 1930, pp. 130 et seq.

Hans Kelsen, Wer soll der Hüter der Verfassung sein?, Rothschild, Berlin, 1931.

Hans Kernbauer / Eduard März / Fritz Weber, „Die wirtschaftliche Entwicklung“, in: Erika Weinzierl / Kurt Skalnik (Hrsg), Österreich 1918-1938. Geschichte der Ersten Republik, Band 1, Styria, Graz, 1983, pp. 343 et seq.

Ulrich Kluge, Der österreichische Ständestaat 1934-1938, Verlag für Geschichte und Politik, Wien, 1984.

Friedrich Koja, „Die rechtlichen und politischen Möglichkeiten des Bundespräsidenten bei der Ernennung der Bundesregierung“, Journal für Rechtspolitik 1994, pp. 175 et seq.

Oskar Lehner, Österreichische Verfassungs- und Verwaltungsgeschichte. Mit Grundzügen der Wirtschafts- und Sozialgeschichte, Trauner Verlag, Linz, 4. Aufl. 2007.

Rudolf Machacek, „80 Jahre B-VG - 80 Jahre VfGH. 150 Jahre RA-Kammer für Wien und NÖ. Aus Anlass des 60. Geburtstages von Univ.-Prof. Dr. Karl Korinek, Vize-Präsident des VfGH," Anwaltsblatt 2000, pp. 652 et seq.

Adolf Merkl, „Zur Verfassungsreform. Die Verfassungsnovelle im Lichte der Demokratie“, Juristische Blätter 1929, pp. 469 et seq.

Adolf Merkl, „Der rechtliche Gehalt der österreichischen Verfassungsreform vom 7. Dezember 1929“, Zeitschrift für öffentliches Recht 1931, pp. 161 et seq.

Wolfgang C Müller, „Austria: The 'Authority in Reserve' Presidency“, in: Robert Elgie (Hrsg), Semi-Presidentialism in Europe, Oxford University Press, Oxford, 1999, pp. 22 et seq. 
Alfred J Noll / Emmerich Tálos, „Streit um Worte oder Streit um die Sache? Zu Ludwig Adamovich” „Das autoritäre System 1933 - 1938 zwischen Rechtsbruch und Gewissensentscheidung ““, Journal für Rechtspolitik 2015, pp. 277 et seq.

Theo Öhlinger, Der Stufenbau der Rechtsordnung, Manz, Wien, 1975.

Theo Öhlinger, Hans Kelsen und das österreichische Bundesverfassungsrecht [Vortrag im Rahmen der Tagung „Die österreichischen Einflüsse auf die Modernisierung des japanischen Rechts“, Wien, 20.3.2003], www.univie.ac.at/staatsrecht-oehlinger/php/get.php?id=143.

Theo Öhlinger / Harald Eberhard, Verfassungsrecht, facultas, Wien, 11. Aufl. 2016.

Theo Öhlinger / Maximilian Steinbeis, „Das wäre wohl so etwas wie eine Verfassungskrise“, VerfBlog, 2016/4/25, https://verfassungsblog.de/das-waerewohl-so-etwas-wie-eine-verfassungskrise/.

Anton Pelinka, „Nicht schon wieder Weimar. Zur Sehnsucht nach einem starken Bundespräsidenten“, Journal für Rechtspolitik 1994, pp. 140 et seq.

Hugo Preuß, Gesammelte Schriften. Band 4, Mohr Siebeck, Tübingen, 2008.

Wolfgang Putschek, Ständische Verfassung und autoritäre Verfassungspraxis in Österreich 1933-1938, Peter Land, Frankfurt am Main, 1993.

Gerhard G Senft, Im Vorfeld der Katastrophe. Die Wirtschaftspolitik des Ständestaates. Österreich 1934-1938, Braumüller, Wien, 2002.

Carl Schmitt, Der Hüter der Verfassung, Mohr, Tübingen, 1931.

Christian Sima, Österreichs Bundesverfassung und die Weimarer Reichsverfassung. Der Einfluss der Weimarer Reichsverfassung auf die österreichische Verfassung 1920 bis 1929, Lang, Frankfurt am Main, 1993.

Walter B Simon, Österreich 1918-1938, Böhlau, Wien, 1984.

Kurt Strele, Rechtsstaat und Demokratie im neuen Österreich, Wagner, Innsbruck, 1931.

Stefanie Vielgut, Demokratie, Verfassung und Rechtspositivismus. Über die Entstehung und den Untergang der Weimarer Republik und der Ersten Republik Österreich, AV Akademiker Verlag, 2012.

Manfried Welan, „Präsidialismus oder Parlamentarismus? Demokratiepolitische Perspektiven“, in: David F. J. Campbell et al. (Hrsg.), Die Qualität der österreichischen Demokratie. Versuche einer Annäherung, Opladen, Wien, 1996, pp. 59 et seq.

Ewald Wiederin, „Österreich“, in: Armin von Bogdandy/Pedro Cruz Villalón/Peter M. Huber (Hrsg.), Ius Publicum Europaeum, Band I, Müller, Heidelberg, 2006, pp. 389 et seq.

Ewald Wiederin, „Denken vom Recht her. Über den modus austriacus in der Staatsrechtslehre", in: Helmuth Schulze-Fielitz (Hrsg.), Staatsrechtslehre als Wissenschaft, Duncker \& Humblot, Berlin, 2007, pp. 293 et seq.

Ewald Wiederin, „Christliche Bundesstaatlichkeit auf ständischer Grundlage: Eine Strukturanalyse der Verfassung 1934“, in: Reiter-Zatloukal ea (Hrsg), Österreich 1933-1938, Böhlau, Wien, 2012, pp. 31 et seq. 
Ewald Wiederin, „Einheitsstaat oder Bundesstaat? Diskurse und Entscheidungen 1945“, in: Martin P Schennah (Hrsg), Rechtshistorische Aspekte des Föderalismus, Verlag Österreich, Wien, 2015, pp. 183 et seq.

Ewald Wiederin, "Die Weimarer Reichsverfassung im internationalen Kontext", in: Horst Dreier / Christian Waldhoff (Hrsg), Das Wagnis der Demokratie. Eine Anatomie der Weimarer Reichsverfassung, Beck, München, 2018, pp. 45 et seq.

Bernd Wieser, „Art 25 B-VG“, in: Karl Korinek / Michael Holoubek et al (Hrsg), Bundesverfassungsrecht, Verlag Österreich, Wien, 1. Lfg. 1999.

David Wineroither, „Bundespräsident und Bundeskanzler: Konsens, Konflikt oder Neutralität?", Österreichisches Jahrbuch für Politik 2006, pp. 603 et seq.

Fecha de envío / Submission date: 29/04/2019

Fecha de aceptación /Acceptance date: 14/05/2019 